\title{
Of exosomes and epididymsomes: how parental metabolism influences an embryos' fate
}

\author{
David F. Albertini ${ }^{1}$
}

Published online: 27 February 2016

(C) Springer Science+Business Media New York 2016

\begin{abstract}
Along with the price of individuality comes the need for better and more means of communication between like and unlike members of a society. Weighing the advantages and disadvantages of being a single cell in a primordial soup, as we envision evolution of primitive life forms to have begun, commands means of sustainability that drive the basic life forces of all living things including their ability to reproduce.

In pondering the most basic of questions having to do with the foundations of human reproduction, JARG seeks to continue the reductionist dialogue from last month's focus on the axoneme as we parade the minute and microscopic involved with novel mechanisms for cell communication. With technology advancing at a mind-boggling pace, elaborating on the ways that our chromosomes and genes can be probed with refinements in molecular methodologies at base pair resolution, the necessity for development of more robust methods of data analysis has had to follow suit. It can be argued that the surge in molecular biology, especially as it applies to genetics, has overshadowed the elucidation of cellular and subcellular activities that play directly into our understanding of the very processes so basic to human reproduction.
\end{abstract}

Communicating information within and between cells is the essence of living matter. It is becoming emblematic in

Capsule Intercellular communication commands a presence in reproductive physiology, and with the emergence of exosomes as conduits for the exchange of small RNAs between cells, laboratory practices for the preparation of gametes and embryos may need to be reconsidered as epigenetic modifiers in the context of human ARTs.

David F. Albertini

DALBERTINI@kumc.edu

1 University of Kansas Medical Center, Kansas, KS, USA reproductive medicine for basic properties such as hormone action, where the trails to patient care become more reliant on the plethora of substances that can be assayed with speed and accuracy to classify patients into categories and monitor their daily rhythms as they move through an ovarian stimulation cycle or await that frozen embryo on the day designated for transfer.

There is no better example than the priming of the ovarian follicle, with growth and steroid output assuming roles as sentinels for that precious cargo within, the oocyte and its granulosa cell investments. As the clinician takes control over the transformation of their previously monovular patient into that of a polyovular one, each bolus of gonadotropin encourages the synthesis and secretion of estrogen through mechanisms that have long been regarded as classical cases of signal transduction through the receptors for FSH and LH presented by granulosa cells. This form of communication is not alone during the pathway to follicle maturation. For decades now, it has been known, but little appreciated, that within the follicle, an extensive network of gap junctions exists to effectively transform the inner doings of a growing follicle into a functional syncytium not unlike that which sustains the beating heart. And superimposed on this gonadotropin-driven mass behavior of cells holding hands has arrived the notion of the many growth factors derived from either the oocyte or granulosa cells that reciprocally modulate the destiny of each to assure that the oocyte remains poised in the meiotic cell cycle to respond to the LH surge while delimiting precocious luteinization of the follicle. Without this programmed behavior of granulosa cells mediated by signals from the oocyte itself, changes in the follicle milieu will adversely affect oocyte quality.

The elaborate and multilayered system of communication pathways that syncytializes the ovarian follicle has just become more complicated based on the discovery of exosomes, 
extracellular vesicles (EVs) produced by many cell types and now recognized as a major form of intercellular crosstalk. While the history and heritage of intracellular vesicles is a long and well-characterized one that rests at the heart of receptor-mediated endocytosis and other internal affairs of the cell, for the EVs their story is just unfolding and will likely have profound implications for human ARTs.

The role of exosomes in the ovary is reviewed this month by Pietro (Exosome-mediated communication in the ovarian follicle. 10.1007/s10815-016-0657-9), and within this article, our readers will find an up-to-date account of ongoing controversies in the identification and characterization of these structures (illustrated on the cover this month). Most importantly, however, Pietro reviews the mounting evidence for RNA cargoes within exosomes that are hypothesized to mediate gene actions between the various cell types of the follicle that have been implicated in either their production or uptake.

The recurrent theme of what the sperm brings to the oocyte to effect its activation is reviewed this month by Anifandis (Sperm contributions to oocyte activation: more than meets the eye 10.1007/s10815-016-0653-0). This subject continues as one of major concerns with patients demonstrating repeated fertilization failure, and as Anifandis points out, our current understanding of the sperm factors responsible for oocyte activation appears to be incomplete and may require serious reinvestigation given two recent publications.

Among the mounting concerns over the procurement and use of human gametes for embryo production has been the issue of what factors may not be coming along for the ride that could impact embryo quality or offspring health. The tale of sperm-associated small RNAs and their possible functions in reproductive health gained significant momentum with the publication of two papers recently in the journal Science. And that we as purveyors and defenders of our collective ARTs should be taking notice has become even more of a pressing issue. These two studies involve the use of mice and, therefore, carry with them the burden of translational relevance to humans, but if the everincreasing "father-load" continues to be documented in mammalian sperm as discussed below, the implications of these findings will have serious ramifications regarding epigenetic disturbances inherent in ARTs.

In the first of these papers, Sharma and colleagues identify a sperm borne small RNA (sRNA) associated with a diet-induced alteration in the metabolism of males so treated that was not present in the sperm of control males (Biogenesis and function of tRNA fragments during sperm maturation and fertilization in mammals. Science. 2016 Jan 22;351(6271):391-6. doi: 10.1126/science. aad6780. Epub 2015 Dec 31). Interestingly, it is shown that sperm acquire this sRNA through their maturation in the epididymis via the sRNA delivered in epididymsomes, the epididymis' version of exosomes. After identifying this as a specific transfer RNA (tRNA) they show that injection of this tRNA into ES cells or embryos leads to repression of endogenous retroelements known to be essential for embryo development.

Following this report in the same issue is the paper by Chen and collaborators who take this discovery alluded to above a step further (Sperm tsRNAs contribute to intergenerational inheritance of an acquired metabolic disorder. Science. 2016 Jan 22;351(6271):397-400. doi: 10.1126/science.aad7977. Epub 2015 Dec 31). Here sperm from males fed a high fat diet were shown to elaborate unique sRNAs relative to controls and upon injection of these into embryos gave rise to offspring bearing the characteristics of metabolic disturbance exhibited by the males from which sperm or sRNAs were derived. This work further evidences the role of paternal epigenetics in offspring health and lends credence to the idea that sperm-borne RNAs impact embryo quality and later development.

That many forms of cell communication are at work simultaneously in the processes that underly human reproduction and development re-emphasizes the importance of redundancy and complexity as applied to the generation of developmentally competent gametes. Bringing exosomes into the limelight that is reproductive biology, gives new meaning to the notion of "reach out and touch someone" with the added implication that it provides a mechanism for our somatic cells (sic selves) to influence the genetics and epigenetics of the developing embryo. 